\author{
Oleksii Doronin $^{*}$, Rostislav Maiboroda ${ }^{*}$
}

\title{
GEE ESTIMATORS IN MIXTURE MODEL WITH VARYING CONCENTRATIONS
}

\begin{abstract}
We discuss a semiparametric mixture model where some components are parameterized with common Euclidean parameter and others are fully unknown. We introduce GEE (generalized estimating equations) approach and adaptive GEE-based approach for parameter estimation. Derived estimators are consistent and asymptotically normal, and they are optimized in terms of their dispersion matrices. Proposed techniques are tested on simulated samples.

Keywords: mixture model, semiparametric estimation, GEE.
\end{abstract}

\section{INTRODUCTION}

The cumulative distribution function (CDF) of one observation in a mixture model is expressed by a linear combination of some CDFs $F_{1}, \ldots, F_{M}$ with probabilities $p^{1}, \ldots, p^{M}, \sum_{m=1}^{M} p^{m}=1$ (i.e. $\left.F_{\xi}(x)=\sum_{m=1}^{M} p^{m} F_{m}(x)\right)$. Note that $F_{m}$ is called the CDF of the $m$-th mixture component, and $p^{m}-$ the component concentration. In mixture model with varying concentrations $p^{m}$ depends on the observation index: $p^{m}=p_{j}^{m}, j=\overline{1, N}$. Thus,

$$
F_{\xi_{j}}(x)=\sum_{j=1}^{M} p_{j}^{m} F_{m}(x), j=\overline{1, N} .
$$

We consider the case when some parametric model is known for the first $K$ components: $F_{m}(x)=F_{m}(x ; t), \quad m=\overline{1, K}$. Parameter $t$ is assumed to be Euclidean: $t \in \Theta \subset \Re^{d}$. The true value of $t$ we designate as $\vartheta$ and assume that it is unknown. The CDFs of the last $M-K$ mixture components are assumed to

* Ph.D. student, Department of Probability Theory, Statistics and Actuarial Mathematics, Mechanics and Mathematics Faculty, Taras Shevchenko National University of Kyiv.

** Ph.D., Department of Probability Theory, Statistics and Actuarial Mathematics, Mechanics and Mathematics Faculty, Taras Shevchenko National University of Kyiv. 
be fully unknown. We also assume that concentrations $p_{j}^{m}$ are known. Our goal is to estimate $\vartheta$. To do this, we derive consistent and asymptotically normal estimators, and optimize them in terms of their dispersion matrices.

\section{NONPARAMETRIC ESTIMATE FOR DISTRIBUTION FUNCTION}

$\mathrm{CDF}$ of the $m$-th component may be estimated through the weighted empirical distribution function:

$$
\hat{F}_{m}(x):=\frac{1}{N} \sum_{j=1}^{N} a_{j}^{m} I_{\left\{\xi_{j} \leq x\right\}} .
$$

Weights $a_{j}^{m}$ are taken as the solution of the minimization problem of maximal variance of unbiased estimates of $F_{m}(x)$ for all possible CDFs $F_{m}$ (i.e. $a^{m}=p \Gamma^{-1} e_{m} \quad$ where $\quad p:=\left(p_{j}^{m}\right)_{j=\overline{1, N}, m=\overline{1, M}} \in \mathfrak{R}^{N \times M}, \quad \Gamma:=\frac{1}{N} p^{T} p \in \mathfrak{R}^{M \times M}$, $\left.e_{m}:=(\chi\{i=m\})_{i=1, \ldots, M}\right)$. See Maiboroda et al. (2008) for details.

Note that weights $a_{j}^{m}$ can be negative. Thus, we can improve $\hat{F}_{m}(x)$ by introducing improved empirical distribution function (see Maiboroda et al. (2005)):

$$
\hat{F}_{m}^{+}(x):=\min \left(1, \max _{y \leq x} F(y)\right) .
$$

\section{GEE ESTIMATE}

Consider some set of measurable functions $g_{1}(\xi ; t), \ldots, g_{K}(\xi ; t) \rightarrow \Re^{d}$. Theoretical moment $\int g_{k}(x ; t) F_{k}(d x)$ may be estimated by the weighted empirical moment as

$$
\hat{g}_{k}^{k}(t):=\frac{1}{N} \sum_{j=1}^{N} a_{j}^{k} g_{k}\left(\xi_{j} ; t\right)
$$

Define the joint weighted empirical moment of $\hat{g}_{k}^{k}(t)$ as

$$
\hat{g}(t):=\sum_{k=1}^{K} \hat{g}_{k}^{k}(t) .
$$


Definition. GEE estimator $\hat{\vartheta}$ for $\vartheta$ is the measurable function from sample $\xi_{1}, \ldots, \xi_{N}$ such that $\hat{g}(\hat{\vartheta})=0$. Next we assume that $P[\exists t \in \Theta: \hat{g}(\hat{\vartheta})=0] \rightarrow 1$ as $N \rightarrow \infty$.

Example. Moment estimators can be represented as GEE estimators. Let $h_{1}, \ldots, h_{K}$ be the set of estimating functions. Denote theoretical moment of $h_{k}(x)$ as $H_{k}(t):=\int h_{k}(x) F_{k}(d x ; t), \quad k=\overline{1, K}$. Define estimating functions as $g_{k}(x ; t):=h_{k}(x)-H_{k}(t), \quad k=\overline{1, K}$. GEE estimator $\hat{\theta}$ can be represented as $\hat{\vartheta}:=H^{-1}\left(\sum_{k=1}^{K} \hat{h}_{k}^{k}\right)$ where $H^{-1}$ is the inversed function to $H(t):=\sum_{k=1}^{K} H_{k}(t)$. Analogous improved moment estimate with $\hat{h}_{k}^{k}:=\int h_{k}(x) \hat{F}_{k}^{+}(d x)$ can be introduced.

Consistency for moment estimators is shown in theorem 3.1 from Doronin (2014a).

\section{ASYMPTOTICS OF GEE ESTIMATOR}

Assume that CDFs $F_{1}, \ldots, F_{M}$ are absolutely continuous with respect to sigma-finite measure $\mu$ on the space of observations. Denote densities of each component's distributions as $f_{k}(x):=\frac{d F_{k}(x ; \vartheta)}{d \mu(x)}, \quad k=\overline{1, K}, \quad f_{k}(x):=\frac{d F_{k}(x)}{d \mu(x)}$, $k=\overline{K+1, M}$.

Introduce the matrix of estimating functions

$$
G(x):=\left(\begin{array}{c}
g_{1}(x ; \vartheta) \\
\vdots \\
g_{K}(x ; \vartheta)
\end{array}\right) \in \mathfrak{R}^{K \times d} .
$$

Expectation of $G(x)$ from the $m$-th component designate as $\bar{G}_{m}:=\int G(x) F_{m}(d x), \quad m=1, \ldots, M$.

Introduce the following notations.

$$
\alpha_{r, s}:=\left(\alpha_{r, s}^{k, l}\right)_{k, l=\overline{1, K}}:=\left(\lim _{N \rightarrow \infty} \frac{1}{N} \sum_{j=1}^{N} a_{j}^{k} a_{j}^{l} p_{j}^{r} p_{j}^{s}\right)_{k, l=\overline{1, K}} \in \mathfrak{R}^{K \times K}, r, s=\overline{1, M}
$$




$$
\begin{gathered}
\beta_{m}:=\left(\beta_{m}^{k, l}\right)_{k, l=\overline{1, K}}:=\left(\lim _{N \rightarrow \infty} \frac{1}{N} \sum_{j=1}^{N} a_{j}^{k} a_{j}^{l} p_{j}^{m}\right)_{k, l=\overline{1, K}} \in \mathfrak{R}^{K \times K}, m=\overline{1, M} . \\
R(x):=\sum_{m=1}^{M} \beta_{m} f_{m}(x) \in \mathfrak{R}^{K \times K} . \\
Z:=\int G(x)^{T} R(x) G(x) \mu(d x)-\sum_{r, s=1}^{M} \bar{G}_{r}^{T} \alpha_{r, s} \bar{G}_{s} \in \mathfrak{R}^{d \times d} . \\
V:=\left.\sum_{k=1}^{K} \int \frac{\partial g_{k}(x ; t)}{\partial t}\right|_{t=9} F_{k}(d x) \in \mathfrak{R}^{d \times d} .
\end{gathered}
$$

Theorem 4.1. (Theorem 3.4 from Doronin (2014a)) Let $\hat{\vartheta}$ be GEE estimator in introduced definitions, and $U$ be some open neighborhood of the true parameter value $\vartheta$. Assume the following.:

(i) $\hat{\vartheta}$ converges in probability to $\vartheta$ as $N \rightarrow \infty$.

(ii) Derivatives $g_{k}^{\prime}(x ; t)=\partial g_{k}(x ; t) / \partial t^{T}$ exist and are integrable (i.e. $\left.E_{t}\left[\left\|g_{k}^{\prime}\left(\eta_{m} ; t\right)\right\|\right]<\infty\right)$ for $t \in U$, where $E_{t}$ denotes expectation under condition that the true parameter value is $t$, and $\eta_{m}$ are the formal random values with distrubutions $F_{m}$.

(iii) Functions $\bar{g}_{k}^{m}(t)=E_{\vartheta}\left[g_{k}\left(\eta_{m} ; t\right)\right]$ are continuous on $U$.

(iv) $E_{\vartheta}\left[\sup _{t \in U}\left\|g_{k}\left(\eta_{m} ; t\right)\right\|\right]<\infty$.

(v) Limit matrix $\Gamma$ exist and is nonsingular.

(vi) Matrices $\alpha_{r, s}$ and $\beta_{m}$ exist.

(vii) Matrix $V$ is nonsingular.

(viii) GEE is unbiased, i.e. $\sum_{k=1}^{K} E_{t}\left[g_{k}\left(\eta_{k} ; t\right)\right]=0$ for $t \in U$.

Then $\sqrt{N}(\hat{\vartheta}-\vartheta)$ converges in distribution to Gaussian distribution with zero mean and covariance matrix $V^{-1} Z V^{-T}$.

\section{LOWER BOUND OF DISPERSION MATRIX FOR GEE ESTIMATOR}

Assume that the matrix $Z$ and nonsingular matrix $V$ exist. Without loss of generality we can assume that two conditions for GEE estimator $\hat{\vartheta}$ are fulfilled:

(i1) $\int g_{k}(x ; \vartheta) F_{k}(d x)=0, k=\overline{1, K}$ (unbiasedness);

(i2) matrix $V$ is the unit matrix. 
Consider the minimization problem of dispersion matrix $Z$ in Loewner ordering (i.e. $A \geq B$ if $A-B$ is non-negatively defined) over all $g_{k}(x ; \vartheta)$ satisfying conditions (i1), (i2). Thus, we have to minimize $c^{T} Z c$ for all $c \in \mathfrak{R}^{d}$. The solution of this problem is the set of estimating functions $g_{k}^{*}(x ; \vartheta)$, which give us the lower bound of dispersion matrix $Z^{*}$ (see theorem 4.1 from Doronin (2014a)).

\section{ADAPTIVE ESTIMATE}

Unfortunately, it is impossible to use in practice the optimal estimating functions $g_{k}^{*}(x ; \vartheta)$, which give the lower bound of dispersion matrix. The first reason is that they depend on unknown densities $f_{k}(x), k=\overline{1, K}$. The second one is the difficulty to solve the GEE in the general case. Therefore, we consider the adaptive approach.

Each function $g_{k}(x ; t)$ can be approximated as $B_{k} u_{k}(x ; t)$ where $B_{k} \in \mathfrak{R}^{d \times L_{k}}$ is some matrix of coefficients to be found, and $u_{k}(x ; t) \in \mathfrak{R}^{L_{k}}$ is the vector of some predefined basis functions (e.g. B-splines). Under conditions (i1), (i2) equation $\sum_{k=1}^{K} \hat{g}_{k}^{k}(t)=0$ we can approximate as

$$
0=\sum_{k=1}^{K} \hat{g}_{k}^{k}(t) \approx \sum_{k=1}^{K} B_{k} \hat{u}_{k}^{k}(\vartheta)+(t-\vartheta) .
$$

The solution of this approximated equation is $t=\vartheta-\sum_{k=1}^{K} B_{k} \hat{u}_{k}^{k}(\vartheta)$. Thus, one can start with some consistent estimate $\widetilde{\vartheta}$ and define adaptive estimate as

$$
\hat{\vartheta}:=\widetilde{\vartheta}-\sum_{k=1}^{K} B_{k} \hat{u}_{k}^{k}(\widetilde{\vartheta})
$$

Consistency and asymptotic normality of introduced adaptive estimate is shown in lemma 3.3 from Doronin (2014b).

\section{NUMERICAL RESULTS}

We chose a three-component mixture model to simulate. All components are taken Gaussian, with parameter values $(m, \sigma)$ as $(-3.2),(3.2),(0.2)$, for each component, respectively. The first two components are assumed to be 
parameterized with $\vartheta=\left(m_{1}, m_{2}, \sigma\right)^{T}$ (different means, common standard deviation). Distribution of the third component is assumed to be fully unknown. Concentrations were also generated as the pseudo-random values, derived by formula $p_{j}^{m}=s_{j}^{m} /\left(s_{j}^{1}+s_{j}^{2}+s_{j}^{3}\right)$ where $s_{j}^{m}$ is taken from uniform distribution on $[0,1]$. Series of samples with sizes 50,100, 250, 500, 750, 1000, 2000, 5000 were simulated, 2000 samples in each series. Vectors of basis functions $u_{k}(x ; t)$ for adaptive estimate were chosen as the set of uniform cubic B-splines with knots at points $m+i \sigma$, where $m$ and $\sigma$ are the mean and standard deviation of the $k$-th component, respectively, $i=-5, \ldots, 5$. Matrices $B_{k}$ were chosen to minimize dispersion matrix. Results are shown in Figure 1.

\section{CONCLUSIONS}

The mixture model with varying concentrations is considered. Several estimators for this model are introduced (moment, GEE, adaptive). The proposed estimators are consistent and asymptotically normal under some conditions. Performance of moment and adaptive estimators are compared on simulated samples. Dispersion of introduced estimators converges to its theoretical asymptotic value for samples with 1000 and more observations.

\section{REFERENCES}

Doronin O. (2014a), Lower bound of dispersion matrix for semiparametric estimation in mixture model. "Theory of Probability and Mathematical Statistics", no. 90, p. 64-76.

Doronin O. (2014b), Adaptive estimation in semiparametric model of mixture with varying concentrations. "Theory of Probability and Mathematical Statistics", no. 91, p. 27-38.

Doronin O. (2012), Robust Estimates for Mixtures with Gaussian Component. "Bulletin of Taras Shevchenko National University of Kyiv. Series: Physics \& Mathematics" (in Ukrainian), vol. 1, p. 18-23.

Maiboroda R., Sugakova O. (2008), Estimation and classification by observations from mixtures. Kyiv University Publishers, Kyiv (in Ukrainian).

Maiboroda R., Kubaichuk O. (2005), Improved estimators for moments constructed from observations of a mixture. "Theory of Probability and Mathematical Statistics", no. 70, p. 83-92.

Maiboroda R., Sugakova O., Doronin A. (2013), Generalized estimating equations for mixtures with varying concentrations. "The Canadian Journal of Statistics", no. 41, vol. 2, p. 217-236. 


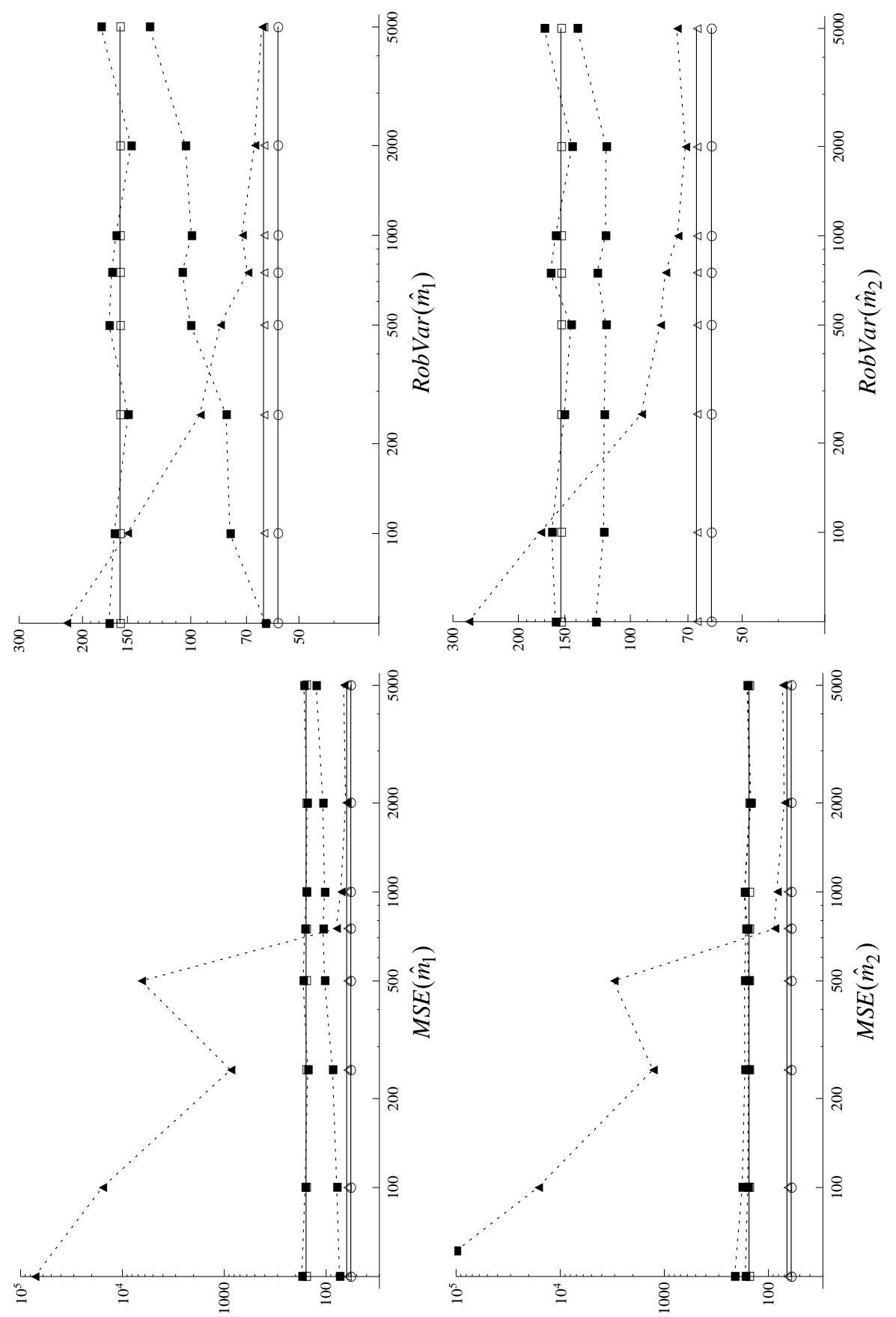




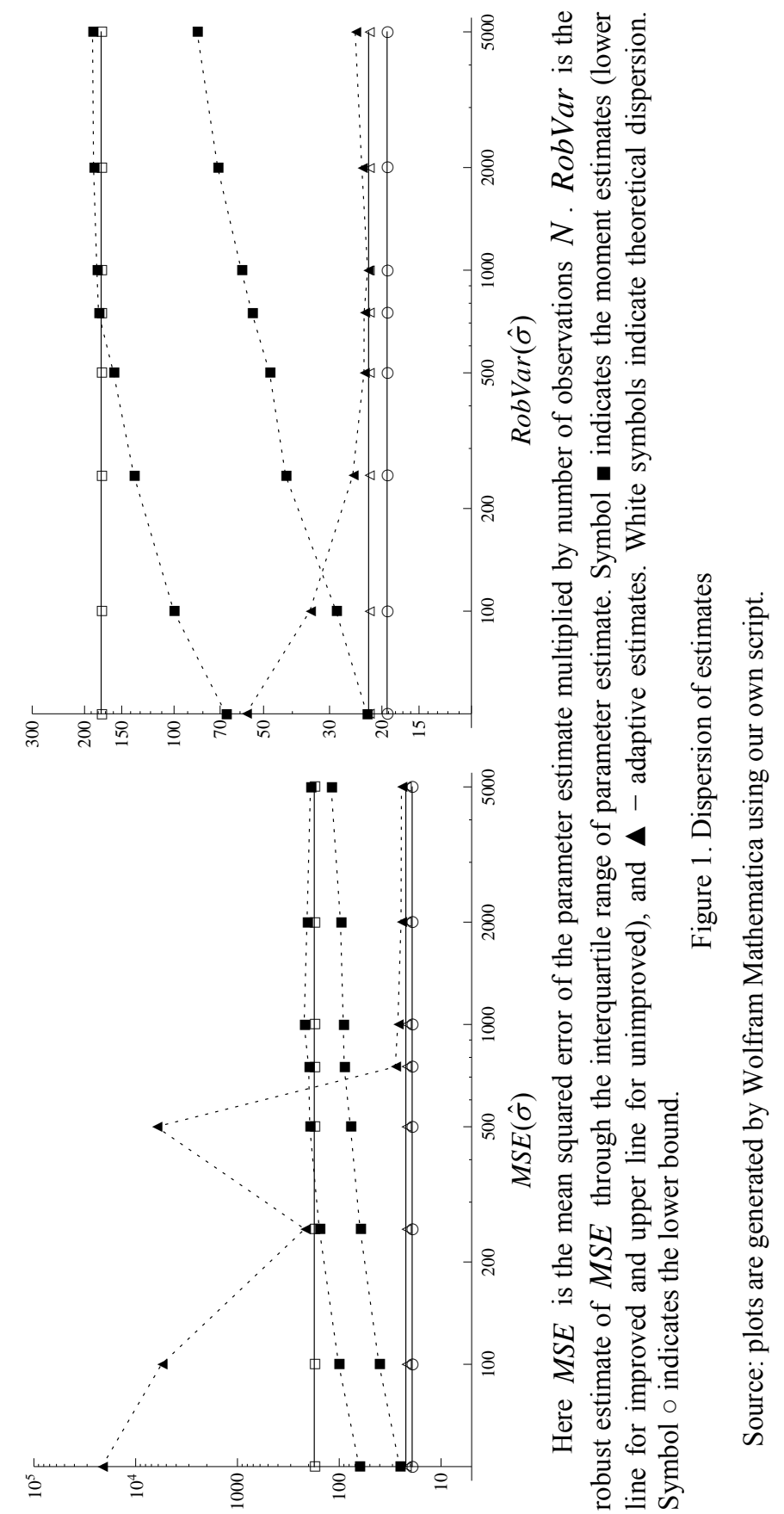


Oleksii Doronin, Rostislav Maiboroda

\section{ESTYMATORY GEE W MODELU MIESZANYM ZE ZMIENNYMI} WSPÓŁCZYNNIKAMI KONCENTRACJI

Streszczenie. W pracy omówiono semiparametryczny model mieszany, w którym pewne współczynniki są parametryzowane za pomocą wspólnego parametru euklidesowego, natomiast inne są zupełnie nieznane. Wprowadzono metodę estymacji parametrów opartą na podejściu GEE (uogólnionych równań estymujących) oraz adaptacyjnym podejściu GEE. Proponowane estymatory zostały przeanalizowane w badaniu symulacyjnym.

Słowa kluczowe: model mieszany, estymacja semiparametryczna, GEE. 
